\title{
Retraction of: "Using the Healthy Days Measure to Assess Factors Associated with Poor Health-Related Quality of Life for Patients with Metastatic Breast, Lung, or Colorectal Cancer Enrolled in a Medicare Advantage Health Plan," by Casebeer AW, et al. Popul Health Manag [e-pub; doi: 10.1089/pop.2018.0024]
}

The article entitled, "Using the Healthy Days Measure to Assess Factors Associated with Poor Health-Related Quality of Life for Patients with Metastatic Breast, Lung, or Colorectal Cancer Enrolled in a Medicare Advantage Health Plan," by Casebeer AW et al, which published online ahead of print in Population Health Management [doi: 10.1089/pop.2018.0024], is being retracted by the authors so that they can remove references to the 8-item Morisky Medication Adherence Scale (MMAS-8) and republish the article without such references.

Dr. Casebeer's team obtained a license to use the MMAS-8 scale from Dr. Morisky prior to conducting the study. Nevertheless, after publication of a separate article in the Journal of Patient-Reported Outcomes that also cited the MMAS8 , as the lead author, Dr. Casebeer was contacted by Dr. Morisky who requested that a number of changes be made to the article. Dr. Casebeer and her team have no desire to engage in a dispute with Dr. Morisky and therefore proactively contacted the Editor of Population Health Management and requested that the scale be removed from her published paper. After assessing all of the provided information, the Editor agreed to the authors' request.

It is important to note that the retraction of Dr. Casebeer's article is not the result of any misconduct on her part or that of her team. The retraction serves to remove the published version of the article that contains the MMAS- 8 scale and a revised version that does not contain the tool or any references to it will be published. The elimination of the scale does not alter the results or conclusions of the study.

Population Health Management is committed to upholding the highest standards of peer review and the community it serves. 